\title{
An fMRI study of joint action-varying levels of cooperation correlates with activity in control networks
}

\author{
Thierry Chaminade ${ }^{1 *}$, Jennifer L. Marchant ${ }^{2,3,4}$, James Kilner ${ }^{5}$ and Christopher D. Frith ${ }^{2,6,7}$ \\ ${ }^{1}$ Institut de Neurosciences de la Timone, Campus Santé Timone, Marseille, France \\ ${ }^{2}$ Wellcome Trust Centre for Neuroimaging, Institute of Neurology, University College London, UK \\ 3 Institute of Cognitive Neuroscience, University College London, UK \\ ${ }^{4}$ Dementia Research Centre, Institute of Neurology, University College London, UK \\ ${ }_{5}^{5}$ Sobell Department of Motor Neuroscience and Movement Disorders, Institute of Neurology, University College London, UK \\ ${ }^{6}$ Interacting minds Group, CFIN, Aarhus University Hospital, Aarhus, Denmark \\ 7 All Souls College, Oxford, UK
}

\section{Edited by:}

Leonhard Schilbach,

Max-Planck-Institute for Neurological

Research, Germany

Reviewed by:

Ivan Toni, Radboud University,

Netherlands

Lisa Aziz-Zadeh, University of

Southern California, USA

Peter H. Weiss, Forschungszentrum

Jülich, Germany

*Correspondence:

Thierry Chaminade, Institut de

Neurosciences de la Timone,

Campus santé Timone,

27 Boulevard Jean Moulin,

Marseille, France.

e-mail: tchamina@gmail.com
As social agents, humans continually interact with the people around them. Here, motor cooperation was investigated using a paradigm in which pairs of participants, one being scanned with $\mathrm{fMRI}$, jointly controlled a visually presented object with joystick movements. The object oscillated dynamically along two dimensions, color and width of gratings, corresponding to the two cardinal directions of joystick movements. While the overall control of each participant on the object was kept constant, the amount of cooperation along the two dimensions varied along four levels, from no (each participant controlled one dimension exclusively) to full (each participant controlled half of each dimension) cooperation. Increasing cooperation correlated with BOLD signal in the left parietal operculum and anterior cingulate cortex (ACC), while decreasing cooperation correlated with activity in the right inferior frontal and superior temporal gyri, the intraparietal sulci and inferior temporal gyri bilaterally, and the dorsomedial prefrontal cortex. As joint performance improved with the level of cooperation, we assessed the brain responses correlating with behavior, and found that activity in most of the areas associated with levels of cooperation also correlated with the joint performance. The only brain area found exclusively in the negative correlation with cooperation was in the dorso medial frontal cortex, involved in monitoring action outcome. Given the cluster location and condition-related signal change, we propose that this region monitored actions to extract the level of cooperation in order to optimize the joint response. Our results, therefore, indicate that, in the current experimental paradigm involving joint control of a visually presented object with joystick movements, the level of cooperation affected brain networks involved in action control, but not mentalizing.

Keywords: fMRI, human neuroscience, joint action, mentalization, motor control

\section{INTRODUCTION}

As social agents, humans continually interact with the people around them. One type of human interaction is cooperation, when two or more people coordinate their actions to achieve a common goal. This is the case when two people carry a heavy object. The joint behavior results from combining the participants' solo actions, and the performance depends on their ability to coordinate these actions. Participants must thus continuously take into account the actions of their partner and adjust their own behavior online accordingly. Each partner is unable to directly control the other's performance, but can influence it for example using with verbal instructions. Such interactivity allows group members to work in synergy as they directly complement each other's performance, even though they may perceive their partner as a hindrance (Reed et al., 2006). Here we investigated a situation in which pairs of participants jointly controlled a visually presented dynamic object using joystick movements, but in addition we manipulated the amount of cooperation while keeping the overall control of each participant constant.

Joint action experiments have provided conflicting results. One experiment demonstrated that dyads provide a better motor control than individuals. When two participants were physically joined to perform a target-reaching task, the behavior of the dyad was improved (i.e., the time to reach the target was reduced) compared to the same participants performing the task alone (Reed et al., 2006). Members of the dyad specialized in their contribution to the task, with one member contributing more to the acceleration and the other to the deceleration. In contrast, another experiment reported degraded tracking performance for pairs of participants that separately controlled the leftward or rightward acceleration of a horizontal marker tracking a target moving on a computer screen, when compared to one participant controlling both directions (Knoblich and Jordan, 2003). 
A recent study of an improvisation game provides possible explanations for this discrepancy. In this game, two players facing each other moved handles on a board to create synchronous, but otherwise unconstrained, motions. There were two conditions: joint improvisation and sequential following. When two experts improvisers performed, their actions were smoother and less jittered when they performed a joint improvisation than when one followed the other. These so-called "moments of togetherness" were captured by a model of mirrored controllers using internal predictions of the other participant's actions, suggesting that jointly improvised action was smoother when both participants anticipated what the other would be doing in the near future (Noy et al., 2011). A study of finger tapping coordination (Konvalinka et al., 2010) similarly concluded that enhanced coordination resulted from the ability of both partners to anticipate the other's actions. Therefore, a working hypothesis is that joint control of an object is improved when each participant in a pair has an internal representation of the other participant's future action. The nature of this internal representation remains unresolved (Seemann, 2011). On the one hand it could pertain only to the overall goal, i.e., the intended behavior of the controlled objects, in which the precise sensorimotor aspects involved in controlling the object would not influence the overall performance. On the other hand, the internal representation could be rooted in embodied motor control systems (Seemann, 2011) in particular in the internal models involved in controlling the self's actions. The sensorimotor aspects of object control would thus play a central role in the behavioral achievements.

To investigate the relative importance of higher order intentions versus lower order sensorimotor integrations in joint actions (Galantucci and Sebanz, 2009), we developed a task in which two players controlled a dynamical object using joystick movements. While the overall goal of the joint action remained constant, the precise sensorimotor transformation required to achieve this goal, in particular the level of cooperation, varied. Most neuroimaging studies of joint action have focused on sequential turn-taking social games (McCabe et al., 2001; Rilling et al., 2002; Sanfey et al., 2003; Decety et al., 2004). A few neuroimaging studies of simultaneous actions constrained participants to act as independent agents working within a pair and investigated the effect of social presence, more than motor cooperation (Sebanz et al., 2006, 2007). There is, however, one study that is particularly relevant to the experiment reported here (Newman-Norlund et al., 2008). fMRI was used to record local brain activity when participants performed a virtual barbalancing task either alone (each hand controlling the either end of the bar) or with a partner. The joint action was further divided into two conditions: each partner controlled one side of the bar, or the two partners shared control over both sides of the bar. The comparison between conditions of shared control and the condition of independent control yielded activity in the right temporoparietal junction (TPJ) and inferior frontal gyrus, regions that can be linked with different aspects of social cognition.

The TPJ and the medial frontal cortex are the two main brain areas involved in mentalizing, i.e., perceiving others' intentions. Although the TPJ responds particularly to the perception of intentional motion (Schultz et al., 2005), the medial frontal cortex responds to a variety of stimuli depicting intentional interactions (see Frith and Frith, 2003 for a review), from intentional animations using simple geometrical shapes (Castelli et al., 2000) to real people acting (Iacoboni et al., 2004). It has been proposed that the medial frontal cortex is involved in the "integration of complex representations of possible actions and anticipated outcomes" (Amodio and Frith, 2006), and it was divided into three functional parts along a rostrocaudal axis: a posterior part involved in monitoring the outcome of action, a middle part involved in representing mental states and an anterior part involved in monitoring of the value of action outcomes (Amodio and Frith, 2006). The TPJ and middle part of the medial prefrontal cortex are expected to be involved when higher-order intentions are being represented internally.

In contrast, the posterior inferior frontal gyrus reported in (Newman-Norlund et al., 2008) is involved in sensorimotor control (Kilner et al., 2009). Particularly interesting is the proposed recruitment of internal models for motor control, which include this area (Kilner et al., 2007), when controlling an object collaboratively. Internal models for the control of action are neural processes that simulate sensorimotor transformations internally, allowing the anticipation of sensory consequences of a planned action (forward models; Wolpert et al., 1995). Internal models have been proposed as possible substrates for social interactions (Wolpert et al., 2003): an agent can use its own forward models when perceiving another agent's actions in order to estimate its hidden mental state. In the case of collaborative control of an object, we propose that internal models are efficient devices for integrating both partners' contribution.

The neural underpinnings of internal models for motor control have been investigated with human non-invasive neuroimaging techniques (for review see Wolpert and Kawato, 1998). Motor commands that are used by forward models to suppress sensory signals are believed to originate upstream from the primary motor cortex (Voss et al., 2006), though they may also involve premotor areas in the posterior inferior frontal gyrus (Kilner et al., 2007). Actual sensory feedback is used to compute prediction errors for model evaluation and update. When we are tickled by another person (Blakemore et al., 1998) the sensory consequences of its actions are unpredictable, and the lack of predictability leads to a high prediction error associated with increased activity in the secondary somatosensory cortex. This area, located bilaterally in the parietal opercula (Eickhoff et al., 2006), plays a key role in sensorimotor integration (Inoue et al., 2002), and has been involved in the assessment of action ownership (Blakemore and Frith, 2003). Accordingly, its activity could be modulated by the level to which members of a group cooperate because disentangling the ownership of complex sensory consequences of a joint action is necessary to improve the individual, and ultimately the joint, motor control. Thus we propose that reliance on internal models for action in the execution of the joint task will be signaled in particular by activity in secondary somatosensory cortices.

The current study aims to identify the brain network in which activity is modulated by the level of group members' cooperation, from fully independent to fully interactive, whilst performing the same joint control task in all experimental conditions. For this 
purpose, pairs of participants were required to control a dynamical object presented visually by tracking changes of a target oscillating along two dimensions (shades of pink and width of gratings) with joystick movements. While both partners always controlled half of the object's overall dynamics, they did so with four different levels of cooperation ranging from no cooperation (one controlled the color, the other the gratings), to full cooperation (each controlled half of the color and half of the gratings). In contrast to the virtual bar-balancing study (Newman-Norlund et al., 2008), the levels of cooperation were graded and implicit. We investigated brain areas in which activity correlates, positively or negatively, with the level of cooperation. Of particular interest is the hypothesis that activity in brain areas involved in mentalizing (in particular the medial prefrontal cortex and TPJ) would correlate with the level of cooperation, suggesting that the scanned participant internal representation of its partner's actions would incorporate its intention to increasingly or decreasingly cooperate. Alternatively, activity associated with the level of cooperation could be limited to brain regions involved in action control (in particular premotor areas, posterior parietal cortex including the secondary somatosensory cortex, cerebellum, and striatum) if collaboration relies exclusively on embodied motor control mechanisms (Seemann, 2011).

\section{METHOD \\ PARTICIPANTS}

Thirteen adult participants (mean age $24+6$ years, seven males) were scanned, eight of these volunteers returned and five additional participants (mean age $26+5$ years, four males) were recruited to take part outside the scanner to form 13 cooperative pairs. All 18 participants were right-handed with no known neurological history and gave informed consent to take part in the study, which was approved by the Joint Ethics Committee of the National Hospital for Neurology and Neuroscience (UCL NHS Trust) and Institute of Neurology (UCL).

\section{EXPERIMENTAL PROCEDURES}

A fixation cross was continuously presented to minimize eye movements. Eye movements were not recorded. A central disk (see Figure 1) was divided into two halves: bottom, the tracking target; top, the joint response. Four rectangles surrounded the circle to indicate the maximal effect for movement in the corresponding cardinal direction-horizontal or vertical. The color dimension ranged from white to red across shades of pink. The width dimension ranged from seven to 10 sinusoid grating cycles (pairs of stripes) across the circle. The circle remained yellow during the $3.2 \mathrm{~s}$ interval between consecutive blocks and participants were asked to let the joystick return to its "neutral" center position. Prior to the start of each block the circle turned to a mid-range color, medium width grating and a $1 \mathrm{~s}$ numerical countdown $(3,2,1)$ was then superimposed. The tracking block commenced when the numbers disappeared and the two dimensions (shades of pink and width of grating) of the target sinusoidally oscillated for four cycles, with a duration of $14.3 \mathrm{~s}$. The duration of a block and the inter-block interval was thus $18.5 \mathrm{~s}$, and 24 blocks were presented in each of the four $9 \mathrm{~min}$ fMRI scanning sessions. The start direction of the target was randomized to prevent anticipation of the response. The relation between the cardinal directions of joystick movements and the dimension of the object under control was counterbalanced between participants.

The scanned player viewed the projected stimuli via a $45^{\circ}$ angled mirror positioned on the head coil while the outside player watched a computer monitor in the scanner control room. Players used their right hand to move a joystick and their left hand to keep it in place during the movements. The joystick movements were recorded and the stimuli were generated and displayed online using Cogent v1.25 (http://www.vislab.ucl.ac.uk/cogent.php) running in Matlab v6.5.1 (The Mathworks, Inc.). Players moved their joysticks to change the properties of the two dimensions (shades of pink and width of grating) of the joint response

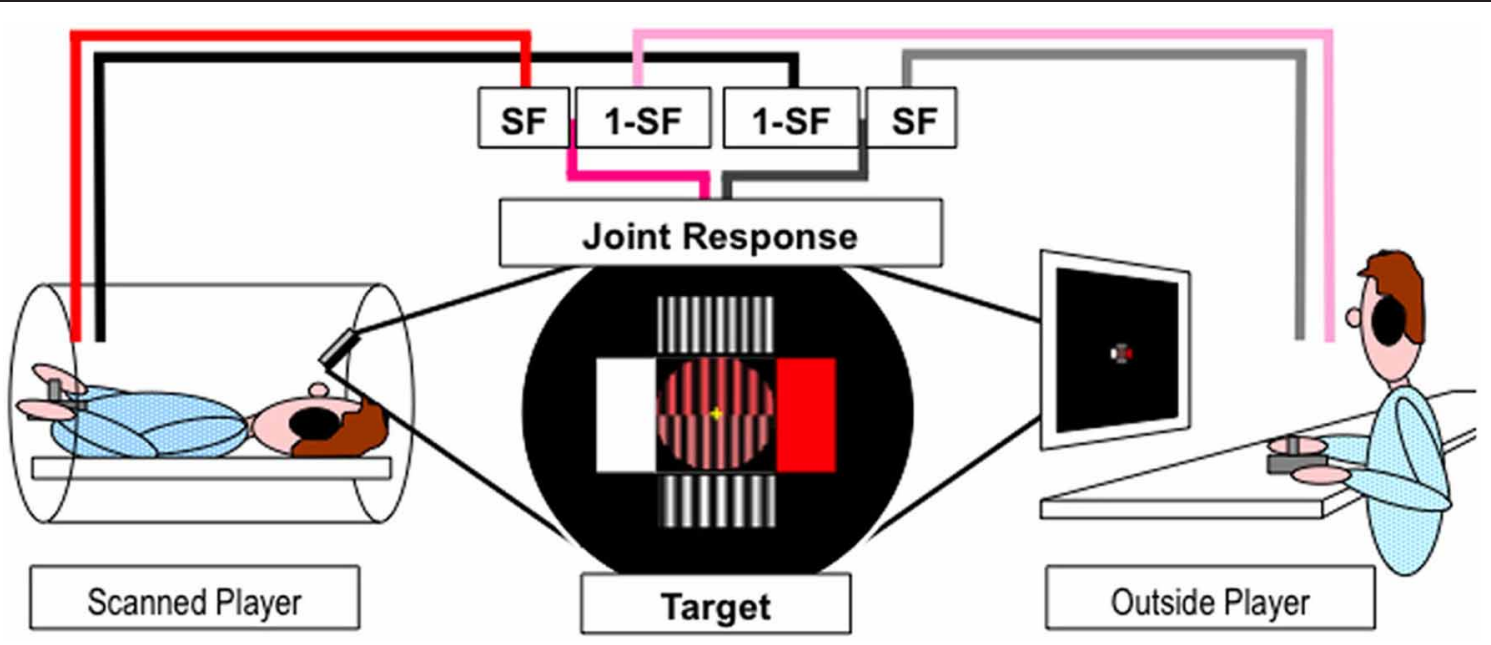

FIGURE 1 | Experimental paradigm. Players inside and outside the scanner used joystick jointly control a dynamic object (stripes of varying width and shades of pink) presented in the upper half of the circle at the center of the screen to match the target presented in the lower half of the circle. Pink/Red and Gray/Black lines represent horizontal and vertical movements respectively. SF represents the scaling factor. 
object to match the target. The change in joystick position in both dimensions were sampled and recorded at $30 \mathrm{~Hz}$ for each player, combined using appropriate cooperation level scaling factor (SF) and summed onto the previous joint response values (see Figure 1). The new joint response and target values were used to generate the new stimuli and refreshed at $30 \mathrm{~Hz}$. Target values, joystick movements and joint response were recorded for behavioral analysis along with time stamps for fMRI analysis.

Participants always retained half control over the whole task, with the other player controlling the other half. However, there were eight conditions, see Table 1, that were split into four different linear levels of cooperation: no cooperation, players fully controlled one dimension ( $\mathrm{SF}=0$ or 1 ); $1 / 3$ cooperation, players controlled the majority of one dimension and a small proportion of the other ( $\mathrm{SF}=0.17$ or 0.83 ); $2 / 3$ cooperation, players controlled double the amount in one dimension than the other ( $S F=0: 33$ or 0.67 ); full cooperation, players equally controlled both dimensions ( $\mathrm{SF}=0.5$ and 0.5 ). Each condition was presented three times per fMRI scanning session in a pseudorandomized order and counterbalanced across pairs. Players were never told explicitly the level of cooperation.

\section{TRAINING AND RECORDING}

At the start of the experiment players were told that their aim was to work together to best match the top to the bottom half of the circle and that the level at which each player controlled the joint response object would vary between blocks, but they would always need to perform joystick movements for successful completion of the task. A training session was performed prior to the scanning day to familiarize themselves with the task and equipment. Players performed a solo practice at the start of both training and scanning days. Each player practiced the task alone following one dimension only (e.g., color), then the other dimension (e.g., grating width) and then tracked both dimensions concurrently with full control over the tracking object. This gradually built the complexity of the task and implicitly demonstrated that the dimensions could be controlled independently. Players were given performance feedback at the end of each solo practice block. On the training day, both players sat at a table in front of individual computer screens separated by a screen and they performed joint control blocks including examples of all the

Table 1 | Level of cooperation scaling factors.

\begin{tabular}{|c|c|c|c|c|c|}
\hline \multirow{3}{*}{$\begin{array}{l}\text { Level of } \\
\text { cooperation }\end{array}$} & \multirow{3}{*}{$\begin{array}{l}\text { Scaling } \\
\text { factor }\end{array}$} & \multicolumn{4}{|c|}{ Factor for each player and dimension } \\
\hline & & \multicolumn{2}{|c|}{ Scanned } & \multicolumn{2}{|c|}{ Outside } \\
\hline & & Color & Width & Color & Width \\
\hline \multirow[t]{2}{*}{0} & 1 & 1 & 0 & 0 & 1 \\
\hline & 0 & 0 & 1 & 1 & 0 \\
\hline \multirow[t]{2}{*}{$1 / 3$} & 0.83 & 0.83 & 0.17 & 0.17 & 0.83 \\
\hline & 0.17 & 0.17 & 0.83 & 0.83 & 0.17 \\
\hline \multirow[t]{2}{*}{$2 / 3$} & 0.67 & 0.67 & 0.33 & 0.33 & 0.67 \\
\hline & 0.33 & 0.33 & 0.67 & 0.67 & 0.33 \\
\hline 1 & 0.50 & 0.50 & 0.50 & 0.50 & 0.50 \\
\hline
\end{tabular}

eight conditions described in Table 1. On the scanning day, players were placed in novel pairs (i.e., never previously experienced played together) with one player lying in the scanner and the other player sat at a computer in the scanner control room. Both players performed the solo practice in situ to familiarize themselves with the environment. Pairs started controlling the object jointly with fMRI scanning, so that they were familiar with the task but had never experienced interacting together before.

\section{MRI DATA ACQUISITION}

A 1.5 T Siemens Sonata MRI scanner (Erlanger, Germany) was used to acquire $\mathrm{T}_{2}^{*}$-weighted gradient echo, echo-planar images with Blood Oxygenation Level-Dependent contrast. Whole brain coverage was achieved using 35 axial slices of $2 \mathrm{~mm}$ thickness with a $2 \mathrm{~mm}$ gap and a TR of $3.15 \mathrm{~s}$. There were four functional imaging runs of 171 volumes and the first six volumes were discarded to allow for effects of $\mathrm{T}_{1}$ equilibrium. An $8 \mathrm{~min} \mathrm{~T}_{1}$-weighted structural MRI scan was acquired for each subject using a hybrid sequence.

Image processing was carried out using Statistical Parametric Mapping software (SPM8; Wellcome Trust Centre for Neuroimaging, www.fil.ion.ucl.ac.uk/spm) implemented in MATLAB v6.5.1. EPI images were realigned to correct for movements, unwarped for motion-induced variance in EPI time-series (Andersson et al., 2001), spatially normalized to standard space using the Montreal Neurological Institute template (voxel size of $2 \times 2 \times 2 \mathrm{~mm}$ ) and spatially smoothed with a $12 \mathrm{~mm}$ full-width half maximum Gaussian kernel.

\section{BEHAVIORAL ANALYSIS}

\section{Joystick movement amplitude}

The amplitude of joystick movements of the scanned player was calculated for each trial. The recorded changes in the joystick position for the two dimensions were combined using the root of the sum of squares to generate the amplitude of movement between successive data point and then summed across the entire block.

\section{Joint performance error}

The joint performance error was calculated to measure how well the players worked together to track the dynamic target and to investigate the effect of the visual feedback of joint performance error on brain activity. The joint response was subtracted from the target for each dimension for each data point and the two dimensions were subsequently combined into a single error measure using the root of the sum of squares to represent the difference between the two halves of the circle. The resulting joint performance error was summed across the entire block.

\section{Behavioral statistical analysis}

Mean joystick movement and joint performance error were averaged across repetitions within each of the four cooperation levels and fMRI scanning session for each participant. These two behavioral measures were entered into separate 4 (levels of cooperation) $\times 4$ (sessions) within-subject repeated measures ANOVAs and significant effects entered into post-hoc pairwise $t$-tests using SPSS. 


\section{MRI STATISTICAL ANALYSIS}

Two first-level fixed-effect analyses were conducted for each subject using the general linear model to separately investigate effect of cooperation and of task performance on BOLD signal. Each first-level design (details below) included condition regressor(s) that comprised $14.3 \mathrm{~s}$ boxcar functions spanning each task performance block convolved with the canonical hemodynamic response function, and six head movement regressors derived from the realignment procedure. Beta estimate contrast images from first-level analyses were entered into second-level random-effects analyses for population inference.

The initial analysis focused on the level of cooperation. Four condition regressors were used to separately model the four levels of cooperation blocks at the first-level. Contrast images corresponding to the main effect of each condition were entered into a second-level random-effects ANOVA of the four levels of cooperation, using the four fMRI scanning sessions as within-subject repeated measures. The four levels of cooperation $(0,1 / 3,2 / 3,1)$ were entered as four dependant levels with equal variance, and contrasts identified the brain correlates of cooperation increase $\left(\left[\begin{array}{llll}-3 & -1 & 1 & 3\end{array}\right]\right)$ and decrease $\left(\left[\begin{array}{llll}3 & 1 & -1 & -3\end{array}\right]\right)$.

The second analysis focused on task performance. A single boxcar function was used to model all four experimental conditions, with a parametric modulator linearly representing the overall joint performance error of each block. Contrast images corresponding to the main effects of the condition regressor and of the parametric regressor were entered into second-level $t$-tests to assess the brain correlates of task execution and of joint performance error respectively.

Results were thresholded at $p<0.05$ corrected for family wise error, extent $k>25$; in the absence of significant results with corrected threholds, the most lenient threshold of $p<0.001$ uncorrected, extent $k>25$ was used, and small volume correction in $10 \mathrm{~mm}$ spherical volume of interest centered on the cluster peak was applied to confirm clusters located in areas of a priori hypotheses survived family wise error correction. Results are reported in MNI space, ordered by decreasing $z$ coordinate.

To further investigate pairwise differences between the levels of cooperation in the medial prefrontal clusters (see results), percent BOLD signal change from all voxels forming the clusters revealed by the whole brain analysis were extracted for each level of cooperation and each fMRI scanning session using MarsBAR (http://marsbar.sourceforge.net/) SPM toolbox implemented in SPM8, and analyzed with 4 (levels of cooperation) $\times 4$ (sessions) ANOVAs using SPSS.

\section{RESULTS \\ BEHAVIORAL RESULTS}

\section{Joystick movement amplitude}

The amplitude of joystick movements made by the scanned player was not significantly modulated by the level of cooperation or the fMRI scanning session (main effects and interaction $p>0.05$ ).

\section{Joint performance error}

Joint performance error was significantly modulated by the level of cooperation $(p<0.001)$, but not by the fMRI scanning session (main effect and interaction with levels of cooperation $p>0.5$ ).

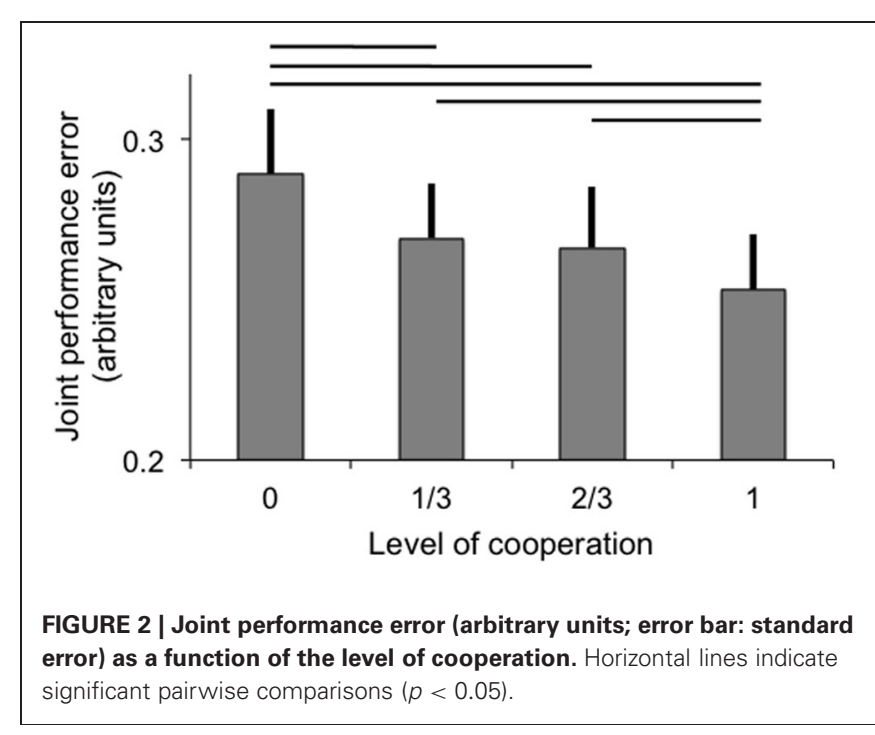

The best joint performance (lowest error) was observed when participants acted in full cooperation and the joint performance error was greatest when participants acted fully independently to control one dimension each (see Figure 2). All pairwise comparisons were significant (at $p<0.05$ ) except between $1 / 3$ and $2 / 3$ levels of cooperation, and a linear fit of the data as a function of the level of cooperation was significant $(p<0.001)$. The effect of sessions and interaction between levels of cooperation and sessions were not significant (both $p>0.1$ ).

\section{fMRI RESULTS}

\section{Level of cooperation}

Firstly, we investigated areas linearly correlated with the four levels of cooperation (contrast $\pm\left[\begin{array}{llll}3 & 1 & -1 & -3\end{array}\right]$ ). In the absence of significant cluster at the threshold used, the more lenient threshold ( $p<0.001$ uncorrected, extent $k>25$ ) was applied (Table 2). Areas positively correlated with the levels of cooperation (see Figure 3) included the left parietal operculum, anterior cingulate cortex (ACC) and hippocampus as well as the cuneus bilaterally. The former two were hypothesized to be involved in cooperation, and small volume correction confirmed they were significantly correlated with cooperation increase (family wise error correction $p=0.003$ in the parietal operculum, $p=0.015$ in the ACC). Areas negatively correlated with the levels of cooperation included the inferior temporal cortex bilaterally, as well as the right superior temporal gyrus and medial prefrontal cortex, and the posterior intraparietal sulcus, right inferior frontal cortex and left cerebellum. The later areas, involved in mentalizing (right superior temporal gyrus and medial prefrontal cortex) and motor control (posterior parietal and premotor cortex and cerebellum) were further explored using small volume correction, yielding significant results (all $p<0.001$ family wise error corrected). Regions involved in visual processing (cuneus and inferior temporal gyrus) and the hippocampus were not further examined in the absence of a priori hypotheses about their involvement in the motor cooperation task.

Percent signal change extracted in the two medial frontal cortex clusters was analyzed using separate repeated-measures 
Table 2 | Brain areas in which activity correlates with levels of cooperation ( $p<0.001$ uncorrected, extent $k>25$ ).

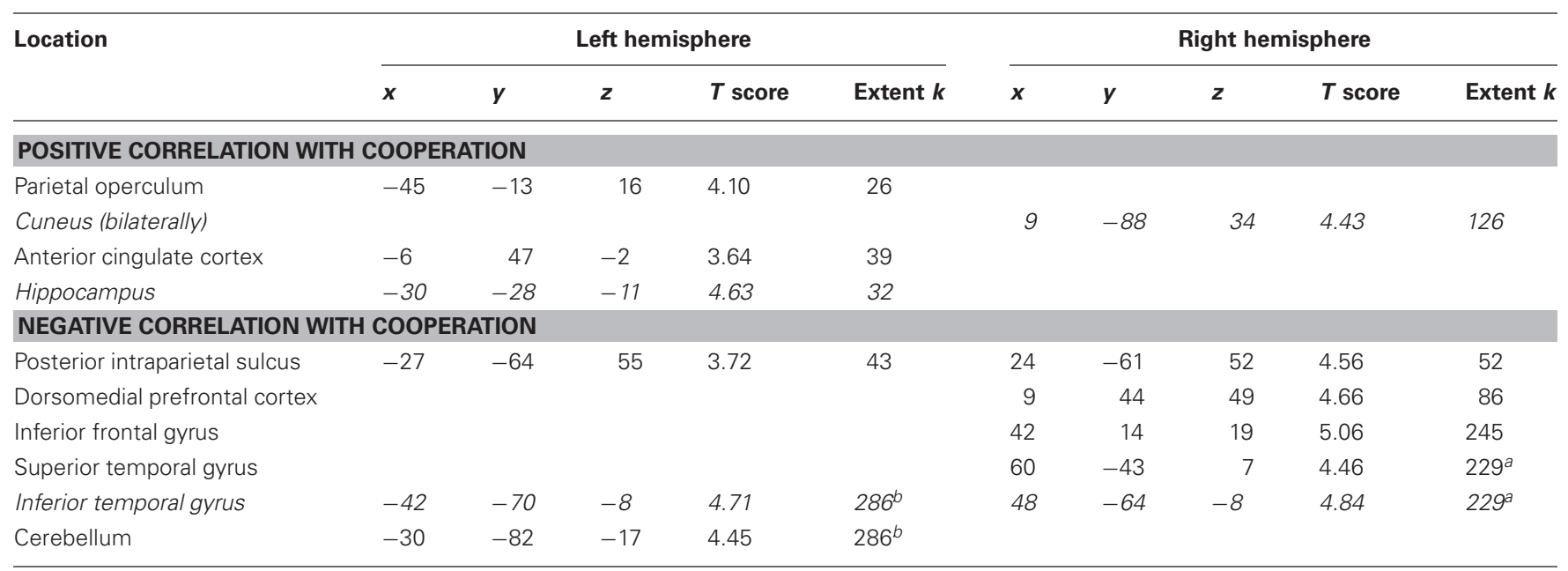

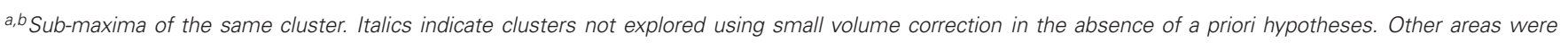
significantly correlated with levels of cooperation.

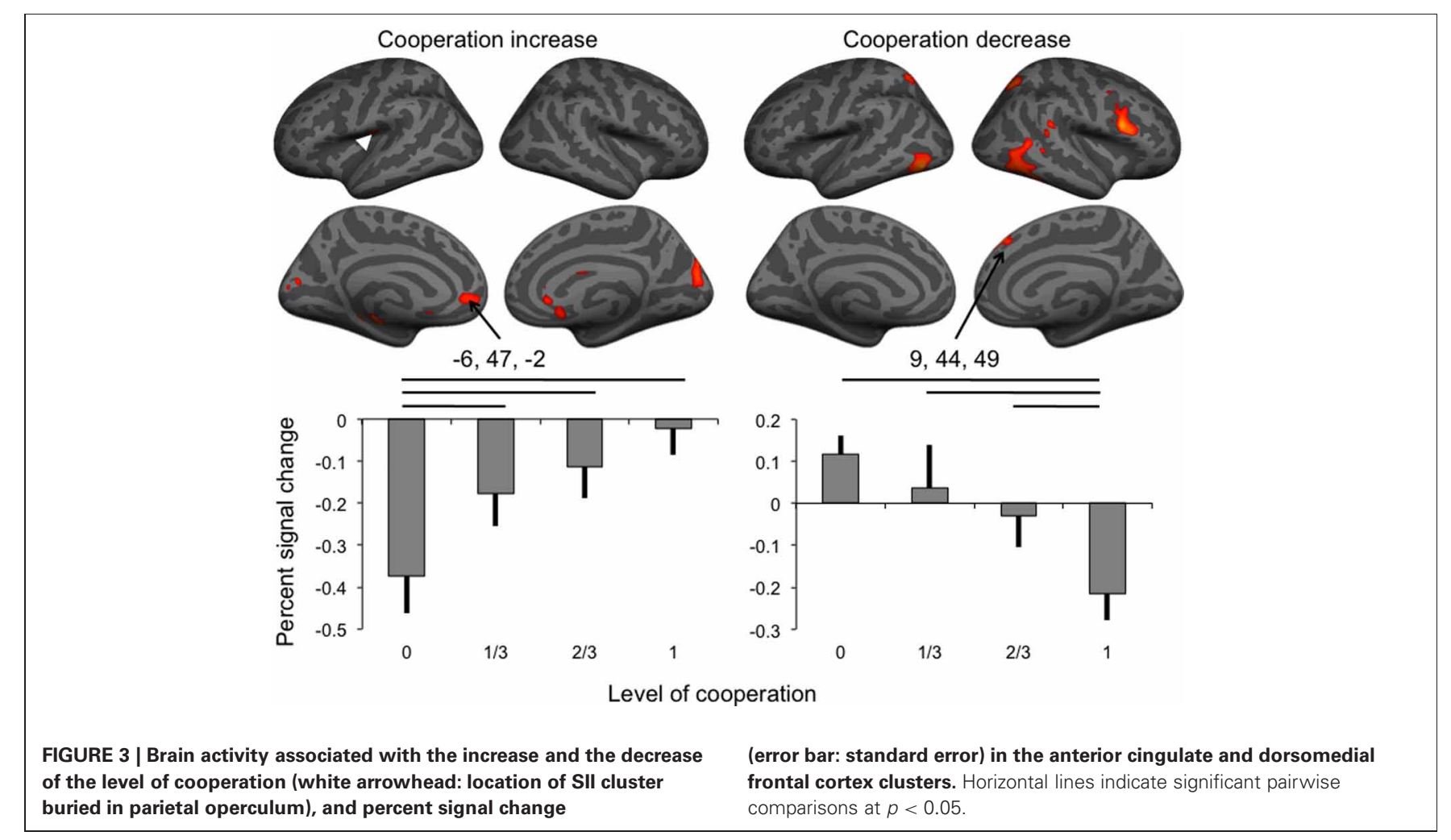

ANOVAs to assess the effect of the levels of cooperation and sessions on percent signal change (Figure 3). For BOLD signal in the dorsal cluster negatively correlated with the level of cooperation, both main effects were significant at $p<0.05$ but not the interaction between the factors $(p=0.747)$; pairwise comparisons indicated that the condition of no cooperation was significantly $(p<0.05)$ different from the three other levels of cooperation, and, as expected from the whole brain analysis, a linear fit of the data as a function of the level of cooperation was significant $(p<0.001)$. For the session factor, the only significant pairwise comparison was between sessions two and three, and a linear fit, corresponding to a decrease of activity with sessions, was significant at $p=0.011$. BOLD signal in the ventral cluster was also significantly affected by the level of cooperation (at $p=0.002$ ) in the absence of any session effect, and pairwise comparisons indicated that the condition of full cooperation was significantly $(p<0.05)$ different from the three other levels of cooperation, and as expected, a linear fit of the 
data as a function of the levels of cooperation was significant $(p=0.002)$.

\section{Task execution}

The main effect of the task execution ( $p<0.05$ corrected for family wise error, extent $k>25$; Table 3 ) was used to identify the sensorimotor network involved in controlling the visually presented object with joystick movements irrespective of the level of cooperation and performance error (Figure 4, left). Right-hand actions on the joystick were associated with left lateralized primary motor cortex, supplementary motor area and putamen, right lateralized dorsal cerebellum activations, and bilateral responses in the middle and inferior occipital gyri, intraparietal sulci extending to the inferior parietal lobes, inferior frontal gyri, frontal eye fields, and ventral cerebellum.

\section{Joint performance error}

Finally, we identified regions positively and negatively correlated with joint performance error ( $p<0.05$ corrected for family wise error, extent $k>25$; Table 4 and Figure 4, right panels). Regions

Table 3 | Main effect of task execution $(p<0.05$ corrected for family wise error, extent $k>25)$.

\begin{tabular}{|c|c|c|c|c|c|c|c|c|c|c|}
\hline \multirow[t]{2}{*}{ Location } & \multicolumn{5}{|c|}{ Left hemisphere } & \multicolumn{5}{|c|}{ Right hemisphere } \\
\hline & $x$ & $y$ & $z$ & $T$ score & Extent $k$ & $\boldsymbol{x}$ & $y$ & $z$ & $T$ score & Extent $k$ \\
\hline Dorsal premotor cortex & -27 & -19 & 73 & 22.01 & $2658^{a}$ & & & & & \\
\hline Primary motor cortex & -39 & -40 & 67 & 33.67 & $2658^{a}$ & & & & & \\
\hline Frontal eye field & -30 & -7 & 64 & 18.12 & $2658^{a}$ & 33 & -7 & 61 & 12.88 & 154 \\
\hline Anterior intraparietal sulcus & -36 & -43 & 58 & 31.00 & $2658^{a}$ & 39 & -43 & 55 & 20.21 & $5216^{b}$ \\
\hline Posterior intraparietal sulcus & -18 & -67 & 52 & 12.86 & $2658^{a}$ & 27 & -58 & 55 & 19.64 & $5216^{b}$ \\
\hline Supplementary motor area & -6 & -19 & 52 & 13.13 & $2658^{a}$ & & & & & \\
\hline Inferior parietal lobule & -57 & -25 & 40 & 25.93 & $2658^{a}$ & 54 & -19 & 31 & 17.39 & $5216^{b}$ \\
\hline Inferior frontal gyrus & -54 & 8 & 22 & 10.98 & $2658^{a}$ & 57 & 8 & 25 & 20.65 & 320 \\
\hline Putamen & -24 & -1 & 1 & 8.99 & 69 & & & & & \\
\hline Middle occipital gyrus & -30 & -91 & -5 & 26.89 & $5216^{b}$ & 33 & -94 & -5 & 24.41 & $5216^{b}$ \\
\hline Inferior occipital gyrus & -48 & -73 & -5 & 17.84 & $5216^{b}$ & 51 & -58 & -8 & 22.42 & $5216^{b}$ \\
\hline Dorsal cerebellum & & & & & & 24 & -49 & -26 & 33.07 & $5216^{b}$ \\
\hline Ventral cerebellum & -15 & -70 & -50 & 17.46 & $5216^{b}$ & 21 & -58 & -47 & 17.08 & $5216^{b}$ \\
\hline
\end{tabular}

a,b Sub-maxima of the same cluster.

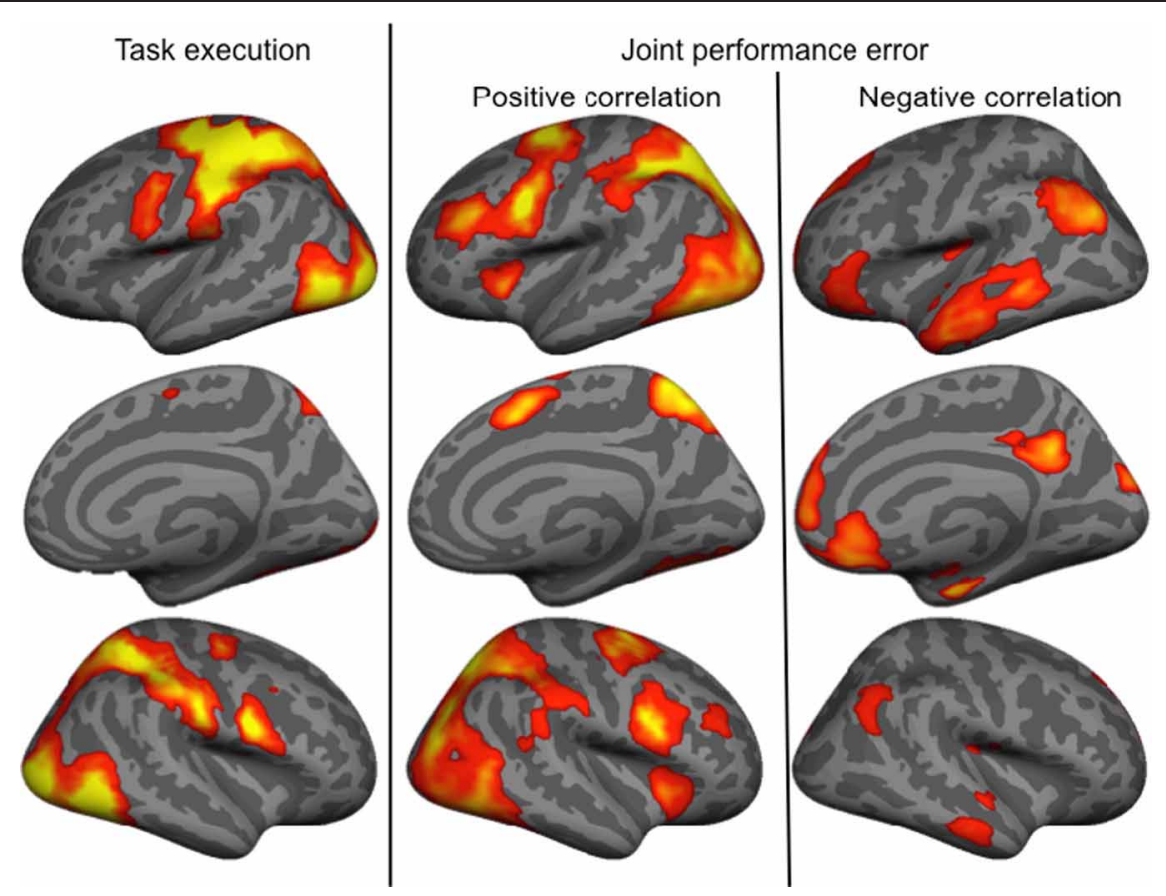

FIGURE 4 | Brain activity associated with the main effect of Task execution, and positively and negatively correlated with the joint performance error 
Table 4 | Brain areas in which activity correlates with joint performance error $(p<0.05$ corrected for family wise error, extent $k>25)$.

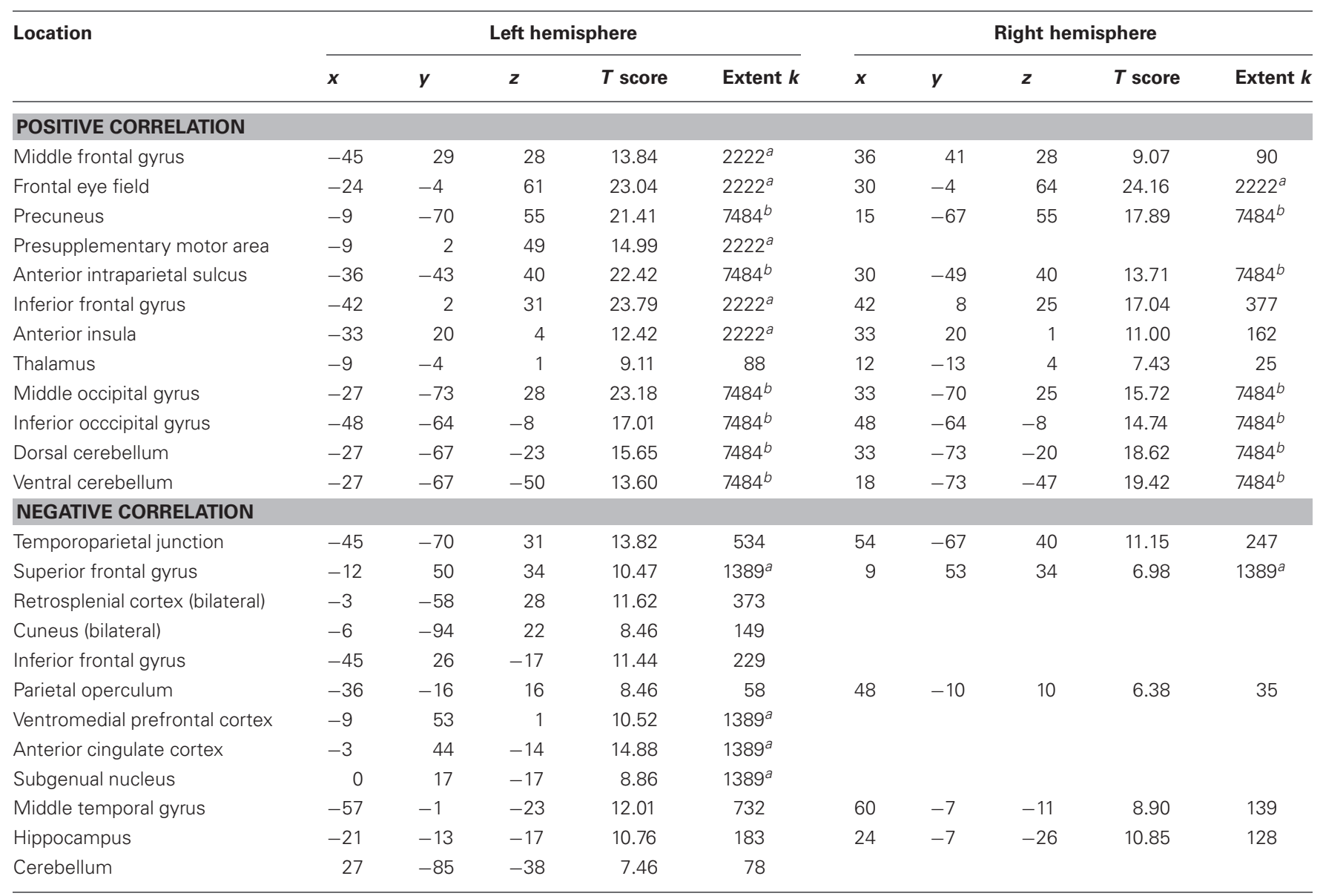

a,b Sub-maxima of the same cluster.

positively correlated with error largely overlapped with the task execution network, including the prefrontal cortex (frontal eye field and inferior frontal gyrus), intraparietal sulcus, cerebellum and lateral occipital cortex, with additional responses in the insula, thalamus, precuneus and middle frontal gyrus bilaterally. The network negatively correlated with the joint performance error, thus positively correlated with the task performance, included the left anterior inferior fontal gyrus, the bilateral TPJ and superior frontal gyrus, anterior lateral and medial (hippocampus) temporal cortex, cuneus, and anterior (ACC and ventromedial prefrontal cortex) and posterior (retrosplenial cortex) regions of the medial wall.

\section{DISCUSSION}

This study investigates how the level of cooperation between two partners modulates brain responses during a joint action task. Pairs of participants manually tracked a two-dimensional visual object with continuous joystick movements and their control over each dimension was manipulated to generate four levels of cooperation ranging from no cooperation (each participant controlled a separate dimension) to full cooperation (both participants controlled half of both dimensions). The overall control over the visual object remained equally shared between the partners.
Behaviorally, joint task performance was improved when cooperation between participants increased. This is in agreement with the finding that partners, haptically connected through handling the two ends of the same manipulandum, showed improved performance in a tracking task when compared to solo performances (Reed et al., 2006). In the current study the no cooperation condition was akin to an individual task in a single dimension, with both participants working in parallel toward mutually exclusive goals. Performance increased gradually, with two out of three pairwise comparisons between contiguous levels of cooperation and a linear fit against the four levels of cooperation statistically significant, so that the improvement could not be solely explained by the main effect of the no cooperation condition: the benefit of cooperation on performance was graded according to degree of cooperation. These results contradict the suggestion that joint actions encounter more coordination problems than individual actions (Knoblich and Jordan, 2003). In Knoblich and Jordan (2003), pairs of participants controlled the velocity of a visually presented object tracking a target toward the right or left of the screen with presses to two buttons, either controlled by one individual (solo condition) or by two individuals (joint action). In contrast, participants in the current experiment moved their joystick continuously and rhythmically. We suggest 
that synchronization with the target was easier when controlling the object position with continuous rhythmic movements, based on central pattern generators, than its velocity with discrete movements, that recruit higher cortical planning areas (Schaal et al., 2004).

Increasing the level of cooperation was associated with BOLD signal in brain regions including the left parietal operculum and ACC. Decreasing cooperation was associated with activity in the right inferior frontal and superior temporal gyri, and bilateral intraparietal sulci. Dissociation was observed in the medial prefrontal cortex, with a positive correlation with increasing cooperation in the ventral ACC and a negative correlation in the dorsomedial prefrontal cortex. Given that task performance was significantly correlated with the level of cooperation, a second analysis of fMRI data focused on brain areas correlated positively or negatively with the performance error to assess whether activated areas associated with levels of cooperation could be attributed to error perception and correction. Almost all regions found activated with increasing collaboration (Figure 3 and Table 2) were indeed also associated with increasing performance (Figure 4 and Table 4). In both the anterior cingulate (see left plot, Figure 3 ) and the secondary somatosensory cortex (white arrow, Figure 3; signal change not shown), the positive correlation with the level of cooperation was mainly driven by a significant reduction of BOLD signal in the condition of no cooperation compared to the other three conditions, with no significant differences between them. The ACC is known to process the outcomes of behaviors in terms of reward (O'Doherty et al., 2001; Amodio and Frith, 2006). For example in an economic game setting, the same area (coordinates $-4,52,-6)$ responded to the probability of obtaining a monetary gain (Knutson et al., 2005). Furthermore it is included in a large cluster positively correlated with joint performance, in agreement with the suggestion that it responds to action outcome, that is, the performance in achieving the joint tracking. Therefore, the positive correlation between activity in the ACC and levels of cooperation is most likely driven with the increase in joint performance with increasing cooperation. Similarly, while not predicted a priori, activity in the anterior hippocampus, that is connected with the anterior cingulate region and plays a role in emotional processing (Fanselow and Dong, 2010), could also reflect the encoding of the reward associated with action outcome.

While the left parietal operculum was associated with cooperation increase, a larger cluster of activity in this region, also reported in the homologous region of the right hemisphere, was found to correlate negatively with joint performance error, suggesting that the computation performed by the parietal operculum was bilateral in our experiment. This region has been associated with internal models for sensorimotor integration of somesthetic consequences of actions. In (Blakemore et al., 1998) this region was activated when participants received a sensory feedback, in that case somatosensory, that did not result from the consequences of their own actions but from an external stimulation. It was proposed that reduction of activity for selfproduced stimuli in the secondary somatosensory cortex, located in the parietal opercula, was related to the cancellation of the sensory consequences of self-generated actions. Here there was a direct correspondence between actions performed along one axis and tracking of the visual object along one dimension in the no cooperation condition. But as a consequence of cooperation increase, the direct effect of the scanned partner's actions on the controlled object were masked by the combination with the effects of the outside partner's actions. Joint performance increase was, therefore, concomitant with a reduction of the correspondence between the action on the joystick and its visible consequences. Reducing predictability of visual input causes increased response in the primary visual cortex (Alink et al., 2010), so that the reduction of predictability of both the color and width of grating dimensions when cooperation increased also accords with the response pattern observed in the cuneus. Results are thus in line with the proposal that the reduced predictability of sensory, in the present case visual, consequences of actions during joint control conditions accounts for the increasing activity in the parietal operculum but also in the cuneus, while the later was not predicted a priori.

Brain regions found associated with decreasing cooperation were also found in the network positively correlated with joint performance error, with the notable exception of the dorsomedial prefrontal cortex discussed later. This implies that their activity must be interpreted in terms of behavior, not of arbitrary levels of cooperation. For instance, the bilateral posterior inferior temporal cortex clusters were located in a ventral stream area involved in high-level visual processing of objects, the lateral occipital complex (Kourtzi and Kanwisher, 2001), and were more likely to respond to the relevant features of the visual input, putatively the comparison between the target and the controlled object indicating the error, than to the levels of cooperation. The right inferior frontal gyrus and left cerebellum are engaged when we use an internal model of a sensorimotor transformation (Imamizu et al., 2007) and were, therefore, involved in online control of joystick movements in response to sensory input. The bilateral posterior intraparietal sulcus clusters are located in regions involved in the control of attention and eye movements (Astafiev et al., 2003) that were required for accurate task performance. The case of the right posterior superior temporal gyrus is particularly interesting because it is spatially close to the TPJ involved in mentalizing. As this region responds to the perception of intentional motions (Schultz et al., 2004), its activity could correspond to the perception of the partner's intentional states. But we didn't expect mentalizing areas to be inversely correlated with levels of cooperation, while the correlation with joint performance error suggests it was involved in the visual perception of the discrepancy between the controlled object and the target. It may be that the perception of error represented a more organic measure of the perceived quality of the cooperation than the arbitrary levels of cooperation.

Results indicate that most clusters in which activity waspositively or negatively-correlated with levels of cooperation were included in the network of brain areas correlatednegatively or positively-with the joint performance error. Positive correlation with joint performance error (Table 4) identified brain areas associated with attention (frontal eye field, intraparietal sulcus; Astafiev et al., 2003) and sensorimotor 
transformation (medial and lateral motor areas; Zhang and Raichle, 2010). Furthermore, it overlapped largely with the brain network involved in task execution, with the absence of the primary motor cortex. Areas correlated with joint performance error but not task execution included the insula that responds to the conscious perception of errors (Ullsperger et al., 2010), and the middle frontal gyrus that intervenes in conscious changes of strategy used to achieve a given task (Badre and D'Esposito, 2009). This finding implies that brain responses to the increase in joint performance error reflect an increased reliance on control mechanisms in the domains of attention, action, and monitoring of errors. In contrast, regions where BOLD signal negatively correlated with joint performance error mapped onto the default mode of brain function (medial orbitofrontal cortex, TPJ, anterior middle temporal gyrus, and retrosplenial cortex bilaterally; Raichle et al., 2001), in line with the anti-correlation between activity in task-positive and task-negative networks (Raichle, 2010). One exception was the precuneus, which belongs to the default mode network but was positively correlated with joint performance error, possibly because of its role in agency: as this region is active when we attribute a perceived (Farrer and Frith, 2002) or imagined (Ruby and Decety, 2001) action to another agent, it is possible that the perceived errors of the controlled object, increasing with cooperation, were attributed to the contribution of the partner outside of the scanner. Altogether, the data indicates that as joint performance error was reduced, when cooperation increased, control resources were freed, and the default mode of brain activity, at the top of the hierarchy between brain systems (Raichle, 2010), took over.

The dorsomedial prefrontal cortex cluster found negatively correlated with cooperation also belongs to the default mode network of brain function (Raichle, 2010). Its reduced response in the full cooperation condition compared to the baseline is thus expected given the motor and attentional demands of the task. But the significant increase of activity of the other conditions doesn't accord with this interpretation given that the task was more demanding in the conditions of partial or no cooperation, as demonstrated by the increase in joint response error. Another interpretation involves participant's strategy for the task, provided that they were not informed of the upcoming condition. In the full cooperation condition the optimal joystick movements followed the diagonal, in the partial cooperation conditions it was angled, and in the no cooperation condition it followed one of the cardinal directions. The optimal strategy, to start with the diagonal, would work for the full cooperation condition, but would become increasingly unfit as cooperation decreases. Accordingly, the dorsomedial prefrontal cortex cluster falls in the posterior region of the rostral medial frontal cortex, involved in monitoring action outcome (Amodio and Frith, 2006). But while the posterior region is involved in monitoring action outcome, activity was not associated with joint performance nor with task execution, even when lenient thresholds of $p<0.001$ uncorrected were used. The data, therefore, suggest that this region participated in monitoring actions outcome not in terms of their absolute error, but in order to extract the hidden experimental variable, namely the level of cooperation corresponding to the joystick movement angles, in order to optimize the joint response. Therefore, the dorsomedial prefrontal cortex activity in relation to the social variable of the task, the level of cooperation, is likely to be associated with motor control.

Considering the original hypothesis, our results indicate that, in the current paradigm, the level of cooperation affected motor control aspects of the task, so that the joint action can be construed as a complex control system in which each participant plays a constitutive role (Seemann, 2011). The absence of mentalizing areas exclusively associated with levels of cooperation could be related to the cooperation paradigm. Firstly, participants were never explicitly told their role within the partnership, or required to make explicit judgments about their partner's performance or intentions, while explicit knowledge about the intentional stance of an interacting partner is known to significantly impact the activity in brain areas involved in mentalizing (Gallagher et al., 2002). Secondly, the only way players could determine their role in the cooperation was by comparing the predicted, but invisible, consequences of their actions to the visible response of the jointly controlled object. Our results suggest that such a computation did not lead to the attribution of increasing intentions to collaborate to the partner. Altogether, it may be that the high motor demands of the task, given the pace of the joystick movements, drove participants to focus on their performance, in particular the discrepancy between the visual feedback expected given the motor command and actually perceived, and to ignore the higher-order computations required to infer the level of cooperation. Thirdly, it was difficult to disentangle brain responses caused by joint performance and by the level of cooperation between players. It may be that in the absence of explicit information about the level of cooperation, the correlation of performance error with brain results represents a more organic measure of perceived cooperation than the arbitrary levels of cooperation we imposed, as discussed in the case of the right posterior superior temporal gyrus. A more ecologically valid joint action paradigm less demanding in terms of motor control and making use of haptic feedback as in the case of joint manipulation (Reed et al., 2006) should be developed to further investigate the interactions between joint actions and mentalizing.

\section{CONCLUSION}

In conclusion, the present investigation of the joint control of a dynamic object participates to the understanding of the cognitive mechanisms underlying joint action. Data confirmed that collaborative action provided a better control than partners playing in isolation. fMRI results suggested that despite its social aspect, the cooperative control of a visually presented object with joystick movements, the task remained primarily a motor control task and we found no evidence that participants had high-order representation of the level of cooperation in the form of neurophysiological correlates of mentalization.

\section{ACKNOWLEDGMENTS}

This work was supported by the Wellcome Trust. Thierry Chaminade received additional support from the ANR (SCAD \#ANR-09-BLAN-0405-02). 


\section{REFERENCES}

Alink, A., Schwiedrzik, C. M., Kohler, A., Singer, W., and Muckli, L. (2010). Stimulus predictability reduces responses in primary visual cortex. J. Neurosci. 30, 2960-2966.

Amodio, D. M., and Frith, C. D. (2006). Meeting of minds: the medial frontal cortex and social cognition. Nat. Rev. Neurosci. 7, 268-277.

Andersson, J. L., Hutton, C. Ashburner, J., Turner, R., and Friston, K. (2001). Modeling geometric deformations in EPI time series. Neuroimage 13, 903-919.

Astafiev, S. V., Shulman, G. L., Stanley, C. M., Snyder, A. Z., van Essen, D. C., and Corbetta, M. (2003). Functional organization of human intraparietal and frontal cortex for attending, looking, and pointing. J. Neurosci. 23, 4689-4699.

Badre, D., and D'Esposito, M. (2009). Is the rostro-caudal axis of the frontal lobe hierarchical? Nat. Rev. Neurosci. 10, 659-669.

Blakemore, S. J., and Frith, C. (2003). Self-awareness and action. Curr. Opin. Neurobiol. 13, 219-224.

Blakemore, S. J., Wolpert, D. M., and Frith, C. D. (1998). Central cancellation of self-produced tickle sensation. Nat. Neurosci. 1, 635-640.

Castelli, F., Happe, F., Frith, U., and Frith, C. (2000). Movement and mind: a functional imaging study of perception and interpretation of complex intentional movement patterns. Neuroimage 12, 314-325.

Decety, J., Jackson, P. L., Sommerville, J. A., Chaminade, T., and Meltzoff, A. N. (2004). The neural bases of cooperation and competition: an fMRI investigation. Neuroimage 23, 744-751.

Eickhoff, S. B., Schleicher, A., Zilles, K., and Amunts, K. (2006). The human parietal operculum. I. Cytoarchitectonic mapping of subdivisions. Cereb. Cortex 16, 254-267.

Fanselow, M. S., and Dong, H.-W. (2010). Are the dorsal and ventral hippocampus functionally distinct structures? Neuron 65, 7-19.

Farrer, C., and Frith, C. D. (2002). Experiencing oneself vs another person as being the cause of an action: the neural correlates of the experience of agency. Neuroimage 15, 596-603.

Frith, U., and Frith, C. D. (2003). Development and neurophysiology of mentalizing. Philos. Trans. R. Soc. Lond. B Biol. Sci. 358, 459-473.
Galantucci, B., and Sebanz, N. (2009). Joint action: current perspectives. Top. Cogn. Sci. 1, 255-259.

Gallagher, H., Jack, A., Roepstorff, A., and Frith, C. (2002). Imaging the intentional stance in a competitive game. Neuroimage 16, 814.

Iacoboni, M., Lieberman, M. D., Knowlton, B. J., Molnar-Szakacs, I., Moritz, M., Throop, C. J., and Fiske, A. P. (2004). Watching social interactions produces dorsomedial prefrontal and medial parietal BOLD fMRI signal increases compared to a resting baseline. Neuroimage 21, 1167-1173.

Imamizu, H., Higuchi, S., Toda, A., and Kawato, M. (2007). Reorganization of brain activity for multiple internal models after short but intensive training. Cortex 43, 338-349.

Inoue, K., Yamashita, T., Harada, T., and Nakamura, S. (2002). Role of human SII cortices in sensorimotor integration. Clin. Neurophysiol. 113, 1573-1578.

Kilner, J., Friston, K., and Frith, C. (2007). Predictive coding: an account of the mirror neuron system. Cogn. Process. 8, 159-166.

Kilner, J. M., Neal, A., Weiskopf, N., Friston, K. J., and Frith, C. D. (2009). Evidence of mirror neurons in human inferior frontal gyrus. J. Neurosci. 29, 10153-10159.

Knoblich, G., and Jordan, J. S. (2003). Action coordination in groups and individuals: learning anticipatory control. J. Exp. Psychol. Learn. Mem. Cogn. 29, 1006-1016.

Knutson, B., Taylor, J., Kaufman, M., Peterson, R., and Glover, G. (2005) Distributed neural representation of expected value. J. Neurosci. 25, 4806-4812.

Konvalinka, I., Vuust, P., Roepstorff, A., and Frith, C. D. (2010). Follow you, follow me: continuous mutual prediction and adaptation in joint tapping. Q. J. Exp. Psychol. (Hove) 63, 2220-2230.

Kourtzi, Z., and Kanwisher, N. (2001). Representation of perceived object shape by the human lateral occipital complex. Science 293, 1506-1509.

McCabe, K., Houser, D., Ryan, L., Smith, V., and Trouard, T. (2001) A functional imaging study of cooperation in two-person reciprocal exchange. Proc. Natl. Acad. Sci. U.S.A. 98, 11832-11835.

Newman-Norlund, R. D., Bosga, J., Meulenbroek, R. G., and Bekkering,
H. (2008). Anatomical substrates of cooperative joint-action in a continuous motor task: virtual lifting and balancing. Neuroimage 41, 169-177.

Noy, L., Dekel, E., and Alon, U. (2011). The mirror game as a paradigm for studying the dynamics of two people improvising motion together. Proc. Natl. Acad. Sci. U.S.A. 108, 20947-20952.

O’Doherty, J., Kringelbach, M. L., Rolls, E. T., Hornak, J., and Andrews, C. (2001). Abstract reward and punishment representations in the human orbitofrontal cortex. Nat. Neurosci. 4, 95-102.

Raichle, M. E. (2010). Two views of brain function. Trends Cogn. Sci. 14 180-190.

Raichle, M. E., MacLeod, A. M., Snyder, A. Z., Powers, W. J., Gusnard, D. A., and Shulman, G. L. (2001) A default mode of brain function. Proc. Natl. Acad. Sci. U.S.A. 98 676-682.

Reed, K., Peshkin, M., Hartmann, M. J., Grabowecky, M., Patton, J., and Vishton, P. M. (2006). Haptically linked dyads: are two motor-control systems better than one? Psychol. Sci. 17, 365-366.

Rilling, J., Gutman, D., Zeh, T., Pagnoni, G., Berns, G., and Kilts, C. (2002). A neural basis for social cooperation. Neuron 35, 395.

Ruby, P., and Decety, J. (2001). Effect of subjective perspective taking during simulation of action: a PET investigation of agency. Nat. Neurosci. 4, 546-550.

Sanfey, A. G., Rilling, J. K., Aronson, J. A., Nystrom, L. E., and Cohen, J. D. (2003). The neural basis of economic decision-making in the Ultimatum Game. Science 300, 1755-1758.

Schaal, S., Sternad, D., Osu, R., and Kawato, M. (2004). Rhythmic arm movement is not discrete. Nat. Neurosci. 7, 1136-1143.

Schultz, J., Friston, K. J., O’Doherty, J., Wolpert, D. M., and Frith, C. D. (2005). Activation in posterior superior temporal sulcus parallels parameter inducing the percept of animacy. Neuron 45, 625-635.

Schultz, J., Imamizu, H., Kawato, M. and Frith, C. D. (2004). Activation of the human superior temporal gyrus during observation of goal attribution by intentional objects. J. Cogn. Neurosci. 16, 1695-1705.

Sebanz, N., Knoblich, G., Prinz, W. and Wascher, E. (2006). Twin peaks: an ERP study of action planning and control in co-acting individuals. J. Cogn. Neurosci. 18, 859-870.

Sebanz, N., Rebbechi, D., Knoblich, G., Prinz, W., and Frith, C. D. (2007). Is it really my turn? An event-related fMRI study of task sharing. Soc. Neurosci. 2, 81-95.

Seemann, A. (2011). Joint motor action and cross-creature embodiment. Rev. Philos. Psychol. 2, 279-301.

Ullsperger, M., Harsay, H. A., Wessel, J. R., and Ridderinkhof, K. R. (2010). Conscious perception of errors and its relation to the anterior insula. Brain Struct. Funct. 214, 629-643.

Voss, M., Ingram, J. N., Haggard, P., and Wolpert, D. M. (2006). Sensorimotor attenuation by central motor command signals in the absence of movement. Nat. Neurosci. 9, 26-27.

Wolpert, D., Doya, K., and Kawato, M. (2003). A unifying computational framework for motor control and social interaction. Philos. Trans. R. Soc. Lond. B Biol. Sci. 358, 593-602.

Wolpert, D. M., Ghahramani, Z., and Jordan, M. I. (1995). An internal model for sensorimotor integration. Science 269, 1880-1882.

Wolpert, D. M., and Kawato, M. (1998). Multiple paired forward and inverse models for motor control. Neural Netw. 11, 1317-1329.

Zhang, D., and Raichle, M. E. (2010). Disease and the brain's dark energy. Nat. Rev. Neurol. 6, 15-28.

Conflict of Interest Statement: The authors declare that the research was conducted in the absence of any commercial or financial relationships that could be construed as a potential conflict of interest.

Received: 29 February 2012; accepted: 31 May 2012; published online: 15 June 2012.

Citation: Chaminade T, Marchant JL, Kilner J and Frith CD (2012) An fMRI study of joint action-varying levels of cooperation correlates with activity in control networks. Front. Hum. Neurosci. 6:179. doi: 10.3389/fnhum.2012.00179 Copyright (c) 2012 Chaminade, Marchant, Kilner and Frith. This is an open-access article distributed under the terms of the Creative Commons Attribution Non Commercial License, which permits non-commercial use, distribution, and reproduction in other forums, provided the original authors and source are credited. 